\title{
DISTRIBUTION OF MASS FOR AVERAGES OF NEWTONIAN POTENTIAL FUNCTIONS
}

BY J. M. THOMPSON

1. Introduction. It has been proved that the average of a potential function over a spherical volume and the average of a potential function over a spherical surface are themselves potential functions.* This paper is concerned with the determination of the distribution of mass for these two spherical averages; in addition, the distribution of mass for more general averages is obtained.

2. Preliminary Theorems. The problem is solved by means of a theorem on the change of the order of integration of an iterated Stieltjes integral. First it is necessary to state some preliminary theorems. We recall the following elementary theorem.

If $h(Q)$ is continuous in $Q$ and $g(e)$ is a distribution of positive mass, bounded in total amount, and lying on a bounded set $F$ (which may be taken as closed without loss of generality), then, for the integral over the whole of space, w,

$$
\left|\int_{w} h(Q) d g\left(e_{Q}\right)-\sum_{w} h\left(Q_{i}\right) g\left(e_{i}\right)\right|<\omega_{\delta} \alpha,
$$

where the summation is extended over all the meshes of a lattice $L_{\delta}$, of diameter $\leqq \delta, Q_{i}$ is a point of the mesh $e_{i}, \omega_{\delta}$ is the oscillation of $h(Q)$ on a subset of $F$ of diameter $\leqq \delta$, and $\alpha \geqq g(F)$.

This theorem will be applied to the integral

$$
\int_{w} h^{N}(M, Q) d g\left(e_{Q}, P\right),
$$

where $h^{N}(M, Q)$ is continuous in $M, Q$, and $g(e, P)$ and $F$ are bounded independently of $P$, so that $\omega_{\delta}$ and $\alpha$ in (1) are independent of $M, P$.

Theorem 1. If $g(e, P)$ is a distribution of positive mass, bounded independently of $P$, on a set $F$ bounded independently

* G. C. Evans, On potentials of positive mass, Transactions of this Society, vol. 37 (1935), p. 250. 
of $P$, and if $f(e)$ is a distribution of positive mass, bounded in all space, and if $g(e, P)$ is summable with respect to $f(e)$, then

$$
G(e)=\int_{w} g(e, P) d f\left(e_{P}\right)
$$

is a distribution of positive mass bounded in total amount and lying on the set $F$.

In this theorem, it is not required that $g(e, P)$ be continuous in $P$, so that the integral must be interpreted as a generalized or Daniell integral with respect to $f(e)$. But in the theorems which follow, $g(e, P)$ is taken as continuous in $P$ for every mesh of $L$.

To prove that $G(e)$ is a distribution of positive mass, we must show that $G(e)$ is a non-negative, absolutely additive function of point sets (only Borel measurable point sets are considered).

The first requirement follows immediately from the fact that $g(e, P)$ and $f(e)$ are non-negative for all $P$ and $e$. In order to exhibit the second condition, let $e=e_{1}+e_{2}+\cdots$ and $e_{n}^{\prime}=e_{1}+e_{2}+\cdots+e_{n} ;$ as $g(e, P)$ is a distribution of positive mass, we have

$$
g(e, P)=\lim _{n=\infty} g\left(e_{n}^{\prime}, P\right), \text { with } g\left(e_{n+1}^{\prime}, P\right) \geqq g\left(e_{n}^{\prime}, P\right) .
$$

Since $g(e, P)$ is, as a function of $P$, the limit of an increasing sequence of functions, the order of integration and passing to the limit may be interchanged; so that

$$
\begin{aligned}
G(e) & =\int_{w} g(e, P) d f\left(e_{P}\right)=\lim _{n=\infty} \int_{w} g\left(e_{n}^{\prime}, P\right) d f\left(e_{P}\right) \\
& =\lim _{n=\infty}\left[G\left(e_{1}\right)+\cdots+G\left(e_{n}\right)\right]=G\left(e_{1}\right)+G\left(e_{2}\right)+\cdots
\end{aligned}
$$

Hence $G(e)$ is a distribution of positive mass. Also $G(e)$ is a distribution of mass lying on $F$; that is, $G(e)=0$, if $e \cdot F=0$, because of the hypothesis that $g(e, P)=0$, if $e \cdot F=0$. Finally, $G(e) \leqq[$ upper bound of $g(e, P)] \cdot f(w)$, so bounded.

We state the generalizations of two theorems proved by $\mathrm{H}$. E. Bray; the proofs are omitted as they are essentially the same as those given by Bray.* They depend on the inequality (1).

* H. E. Bray, Elementary properties of the Stieltjes integral, Annals of Mathematics, vol. 20 (1918-19), pp. 180-185. 
THEOREM 2. If $h^{N}(M, Q)$ is a continuous function of $M$ and $Q$, and is bounded in all space, if $g(e, P)$ is a distribution of positive mass, bounded independently of $P$, on a set $F$ bounded independently of $P$, and if $g(e, P)$ is continuous in $P$ for every cell $e$ of a net $L$, then

$$
K(M, P)=\int_{w} h^{N}(M, Q) d g\left(e_{Q}, P\right)
$$

is continuous in $M$ and $P$.

Theorem 3. If $h^{N}(M, Q)$ is continuous in $M$ and $Q$, and bounded in all space, if $g(e, P)$ is a distribution of positive mass, bounded independently of $P$, on a set $F$ bounded independently of $P$, and if it is continuous in $P$ for every cell e of a net $L$, and if $f(e)$ is a distribution of positive mass, bounded in total amount, lying on a bounded set $E$ (which may be taken as closed without loss of generality), then the integrals

$$
\int_{w} d f\left(e_{P}\right) \int_{w} h^{N}(M, Q) d g\left(e_{Q}, P\right),
$$

and

$$
\int_{w} h^{N}(M, Q) d\left[\int_{w} g\left(e_{Q}, P\right) d f\left(e_{P}\right)\right]
$$

exist and are equal.

We may now state and prove the concluding theorem in this series.

TheORem 4. If $g(e, P)$ is a positive distribution of mass, bounded in total amount independently of $P$, on a set $F$ bounded independently of $P$, and if it is continuous in $P$ for every cell $e$ of the net $L$, and if $f(e)$ is a distribution of positive mass, bounded in total amount and lying on the set $E$, then

$$
\int_{w} d f\left(e_{P}\right) \int_{w} \frac{1}{M Q} d g\left(e_{Q}, P\right)=\int_{w} \frac{1}{M Q} d\left[\int_{w} g\left(e_{Q}, P\right) d f\left(e_{P}\right)\right],
$$

(or both are $+\infty)$, where $\int_{w} g(e, P) d f\left(e_{P}\right)$ is a bounded distribution of positive mass lying on the set $F$. 
If

$$
\begin{aligned}
h^{N}(M, Q) & =\frac{1}{M Q}, \text { if } \frac{1}{M Q} \leqq N, \\
& =N, \quad \text { if } \frac{1}{M Q}>N,
\end{aligned}
$$

the function $h^{N}(M, Q)$ is continuous in $M$ and $Q$.

By Theorem 3, we have

$$
\begin{aligned}
\int_{w} d f\left(e_{P}\right) \int_{w} h^{N}(M, Q) & d g\left(e_{Q}, P\right) \\
= & \int_{w} h^{N}(M, Q) d\left[\int_{w} g\left(e_{Q}, P\right) d f\left(e_{P}\right)\right] .
\end{aligned}
$$

Let $N$ become infinite; we have

$$
\begin{aligned}
\int_{w} d f\left(e_{P}\right) \lim _{n=\infty} \int_{w} h^{N}(M, Q) d g\left(e_{Q}, P\right) \\
=\int_{w} \frac{1}{M Q} d\left[\int_{w} g\left(e_{Q}, P\right) d f\left(e_{P}\right)\right] .
\end{aligned}
$$

The interchange of integration and passing to the limit in the left-hand member is justified because the integrand is an increasing function of $N$, while the definition of the integral of a lowersemi-continuous function with respect to a distribution of positive mass was used in the right-hand member. Applying this definition now to the left-hand member, we have

$$
\int_{w} d f\left(e_{P}\right) \int_{w} \frac{1}{M Q} d g\left(e_{Q}, P\right)=\int_{w} \frac{1}{M Q} d\left[\int_{w} g\left(e_{Q}, P\right) d f\left(e_{P}\right)\right] .
$$

This, combined with Theorem 1, establishes the theorem.

3. Volume Averages. This theorem just proved enables one to exhibit the distribution of mass for the spherical volume average of a potential function in a form in which it may be evaluated; this is an illustration of the advantage sometimes gained by working with the more general situation.

In the theorems that follow, $u(Q)$ is the potential at $Q$ of the distribution of positive mass, $f(e)$, which is bounded in total amount and lies on a bounded set $E$. 
TheOREM 5. The average of the potential function $u(Q)$ over a spherical volume $\Gamma(r, M)$, of radius $r$ and center $M$, is a potential function of a distribution of positive mass with density $\left(3 /\left(4 \pi r^{3}\right)\right) f\{\Gamma(r, Q)\}$. In symbols,

$$
\begin{aligned}
a_{u}(r, M) & =\frac{3}{4 \pi r^{3}} \int_{\Gamma(r, M)} u(Q) d Q \\
& =\frac{3}{4 \pi r^{3}} \int_{w} \frac{1}{M Q} f\{\Gamma(r, Q)\} d Q
\end{aligned}
$$

Let us form this spherical volume average and determine its distribution of mass. We have

$$
\begin{aligned}
a_{u}(r, M) & =\frac{3}{4 \pi r^{3}} \int_{\Gamma(r, M)} u(Q) d Q \\
& =\frac{3}{4 \pi r^{3}} \int_{\Gamma(r, M)} d Q \int_{w} \frac{1}{P Q} d f\left(e_{P}\right)
\end{aligned}
$$

As the integrand is a lower-semi-continuous function, the order of integration may be interchanged, so that

$$
a_{u}(r, M)=\frac{3}{4 \pi r^{3}} \int_{w} d f\left(e_{P}\right) \int_{\Gamma(r, M)} \frac{1}{P Q} d Q .
$$

The inner integral is the potential at $P$ of a sphere of unit density with radius $r$ and center at $M$, which is equal to the potential at $M$ of the same sphere with center at $P$. Hence,

$$
\begin{aligned}
a_{u}(r, M) & =\frac{3}{4 \pi r^{3}} \int_{w} d f\left(e_{P}\right) \int_{\Gamma(r, P)} \frac{1}{M Q} d Q \\
& =\frac{3}{4 \pi r^{3}} \int_{w} d f\left(e_{P}\right) \int_{w} \frac{1}{M Q} d g\left(e_{Q}, P\right),
\end{aligned}
$$

where $g(e, P)=m_{3}\{e \cdot \Gamma(r, P)\}$, and $m_{3}$ means the three-dimensional measure of the set indicated. The quantity $g(e, P)$ is evidently continuous in $P$ for every measurable set $e$.

By means of Theorem 4, this volume average may be expressed in a form which enables the mass distribution to be evaluated, 


$$
a_{u}(r, M)=\frac{3}{4 \pi r^{3}} \int_{w} \frac{1}{M Q} d\left[\int_{w} m_{3}\left\{e_{Q} \cdot \Gamma(r, P)\right\} d f\left(e_{P}\right)\right] .
$$

Consider the integral,

$$
v(e)=\int_{w} m_{3}\{e \cdot \Gamma(r, P)\} d f\left(e_{P}\right),
$$

where $e$ is an arbitrary bounded set measurable Borel. This function is an absolutely continuous function of $e$; in fact

$$
v(e) \leqq m_{3}(e) \cdot f(E),
$$

and $v(e)$ is completely additive by Theorem 1 .

The integrand of $v(e)$ may be expressed as a Lebesgue integral,

$$
m_{3}\{e \cdot \Gamma(r, P)\}=\int_{e \cdot \Gamma(r, P)} 1 d R=\int_{e} B(R, P) d R,
$$

where we may define $B(R, P)$ as 1 , for $R P<r$, and 0 , for $R P \geqq r$. In this way $B(R, P)$ is the limit of an increasing sequence of continuous functions of $R$ and $P$, and we have

$$
\begin{aligned}
v(e) & =\int_{w} d f\left(e_{P}\right) \int_{e} B(R, P) d R=\int_{e} d R \int_{w} B(R, P) d f\left(e_{P}\right) \\
& =\int_{e} f\{\Gamma(r, R)\} d R .
\end{aligned}
$$

Substituting this result in (3) and making use of the definition of the integral of a lower-semi-continuous function, we have

$$
\begin{aligned}
a_{u}(r, M) & =\frac{3}{4 \pi r^{3}} \int_{w} \frac{1}{M Q} d\left[\int_{e_{Q}} f\{\Gamma(r, R)\} d R\right] \\
& =\frac{3}{4 \pi r^{3}} \lim _{N=\infty} \int_{w} h^{N}(M, Q) d\left[\int_{e_{Q}} f\{\Gamma(r, R)\} d R\right] .
\end{aligned}
$$

As $h^{N}(M, Q)$ is bounded and continuous, and $v(e)$ is absolutely continuous, we may change the Stieltjes integral to a Lebesgue integral. This gives

$$
a_{u}(r, M)=\frac{3}{4 \pi r^{3}} \lim _{N=\infty} \int_{w} h^{N}(M, Q) f\{\Gamma(r, Q)\} d Q
$$




$$
=\frac{3}{4 \pi r^{3}} \int_{w} \frac{1}{M Q} f\{\Gamma(r, Q)\} d Q,
$$

and the proof is complete.

It should be pointed out that the work of this section holds for the average over any three-dimensional open set. Let $s(O)$ be an open set (therefore of positive spatial measure), $s(M)$ the set obtained by displacement of $s(O)$ as a rigid body, without rotation, so that $O$ falls on $M$, and let $s^{\prime}(Q)$ be the reflection of the set $s(M)$ through the midpoint of the line $M Q$.

THEOREM 6. The average of the potential function $u(Q)$ over the set $s(M)$ is a potential function of a distribution of positive mass with density $\left(1 /\left[m_{3} s(M)\right]\right) f\left\{s^{\prime}(Q)\right\}$. In symbols,

$$
\begin{array}{r}
a_{u}\{s(M)\}=\frac{1}{m_{3}\{s(M)\}} \int_{s(M)} u(Q) d Q=\frac{1}{m_{3}\{s(M)\}} \\
\int_{w} \frac{1}{M Q} f\left\{s^{\prime}(Q)\right\} d Q .
\end{array}
$$

The construction of the set $s^{\prime}(P)$ gives the potential at $P$ of the set $s(M)$, of unit density, equal to the potential at $M$ of the set $s^{\prime}(P)$, of unit density; hence all the transformations made in this section on the spherical volume average are valid for the average over the set $s(M)$.

4. Spherical Surface Average. By means of Theorem 4, we may also determine the mass function for the average of a potential function over a spherical surface.

THEOREM 7. The average of the potential function $u(Q)$ over the spherical surface $C(r, M)$, of radius $r$ and center $M$, is a potential function of the distribution of positive mass,

In symbols,

$$
\frac{1}{4 \pi r^{2}} \int_{m} m_{2}\{e \cdot C(r, P)\} d f\left(e_{P}\right)
$$

$$
\begin{aligned}
A_{u}(r, M) & =\frac{1}{4 \pi r^{2}} \int_{C(r, M)} u(Q) d Q \\
& =\frac{1}{4 \pi r^{2}} \int_{w} \frac{1}{M Q} d\left[\int_{w} m_{2}\left\{e_{Q} \cdot C(r, P)\right\} d f\left(e_{P}\right)\right]
\end{aligned}
$$


Treating the spherical surface average in the same manner as we have treated the volume average, we obtain

$$
\begin{aligned}
A_{u}(r, M) & =\frac{1}{4 \pi r^{2}} \int_{C(r, M)} u(Q) d Q=\frac{1}{4 \pi r^{2}} \int_{C(r, M)} d Q \int_{w} \frac{1}{Q P} d f\left(e_{P}\right) \\
& =\frac{1}{4 \pi r^{2}} \int_{w} d f\left(e_{P}\right) \int_{C(r, M)} \frac{1}{Q P} d Q \\
& =\frac{1}{4 \pi r^{2}} \int_{w} d f\left(e_{P}\right) \int_{C(r, P)} \frac{1}{Q M} d Q \\
& =\frac{1}{4 \pi r^{2}} \int_{w} d f\left(e_{P}\right) \int_{w} \frac{1}{M Q} d g\left(e_{Q}, P\right),
\end{aligned}
$$

where $g(e, P)=m_{2}\{e \cdot C(r, P)\}$. For a given $P$, this function is additive and bounded for cells $e$ of a three-dimensional lattice, and hence can be extended by definition uniquely to all sets spatially measurable Borel.

As $g(e, P)$ is a continuous function of $P$ for every cell $e$ of a net $L$, Theorem 4 applies, and we may thereby express this average in a form that exhibits its mass function in terms of $C(r, P)$ and $f(e)$,

$$
A_{u}(r, M)=\frac{1}{4 \pi r^{2}} \int_{w} \frac{1}{M Q} d\left[\int_{w} m_{2}\left\{e_{Q} \cdot C(r, P)\right\} d f\left(e_{P}\right)\right] .
$$

This result requires no restriction on $f(e)$ other than those we have already stated. However, we shall state also a special case of Theorem 7.

THEOREM 8. If $f(\Gamma(r, Q)\}$ is differentiable with respect to $r$, and, for a fixed neighborhood of the given value of $r, \partial f\{\Gamma(n, Q)\} / \partial r$ is bounded independently of the point $Q$, then the average of the potential function $u(Q)$ over the spherical surface $C(r, M)$ is a potential function of a distribution of positive mass with density $\left(1 /\left(4 \pi r^{2}\right)\right) \partial f\{\Gamma(r, Q)\} / \partial r$. In symbols,

$$
A_{u}(r, M)=\frac{1}{4 \pi r^{2}} \int_{w} \frac{1}{M Q} \frac{\partial f\{\Gamma(r, Q)\}}{\partial r} d Q
$$

We have, for a rectangular cell $e$,

$$
m_{2}\{e \cdot C(r, P)\}=\lim _{i=\infty} \frac{m_{3}\left\{e \cdot\left[\Gamma\left(r_{i}, P\right)-\Gamma(r, P)\right]\right\}}{r_{i}-r},
$$

where $r<r_{i+1}<r_{i}$ and $\lim _{i=\infty} r_{i}=r$. 
Using the results given in (4), we have the following equality,

$$
\begin{aligned}
\int_{w} \frac{m_{3}\left\{e \cdot\left[\Gamma\left(r_{i}, P\right)-\Gamma(r, P)\right]\right\}}{r_{i}-r} d f\left(e_{P}\right) \\
\quad=\int_{e} \frac{f\left\{\Gamma\left(r_{i}, Q\right)\right\}-f\{\Gamma(r, Q)\}}{r_{i}-r} d Q .
\end{aligned}
$$

The integrand of the left-hand member belongs to a sequence of measurable, uniformly bounded functions, as a function of $P$, whose limit exists when $i$ becomes infinite; so we let $i$ become infinite and interchange the order of integration and pass to the limit for the left-hand member. The same considerations hold for the integrand of the right-hand member as a function of $Q$. Using (13), we have

$$
\int_{w} m_{2}\{e \cdot C(r, P)\} d f\left(e_{P}\right)=\int_{e} \frac{\partial f\{\Gamma(r, Q)\}}{\partial r} d Q .
$$

The quantity $\partial f\{\Gamma(r, Q)\} / \partial r$ is non-negative. Hence we may substitute this last equation in (5) and change the Stieltjes integral into a Lebesgue integral as we did above for the volume average. Thus we have established the theorem.

The University of California

\section{ERRATUM}

In my paper entitled On the summability of a certain class of series of Jacobi polynomials (this Bulletin, vol. 41 (1935), pp. 541-549), the following change should be made; it conforms with the last proofs that I had seen.

Page 544, 8th line from the bottom: read $S_{n, h}^{(k)}$ instead of $S_{n, k}^{(k)}$.

A. P. Cowgir L 